\title{
Defense and counter-defense in rice-virus interactions
}

\author{
Jiaqi Qin, Ci Wang, Leqi Wang, Shanshan Zhao* and Jianguo Wu ${ }^{*}$
}

\begin{abstract}
Rice viruses, known as "rice killer", are vector-borne pathogens that cause severe disease and significant yield loss in rice production around the world. Rice virus disease is characterized by uncontrolled virus replication and the activation of host responses that contribute to pathogenesis. Underlying these phenomena is the potent suppression of rice antiviral responses, particularly the RNA silencing pathway and plant hormone pathways, which play vital roles in antiviral immunity. Classical rice virus disease control strategies include chemotherapeutics and use of disease resistance rice varieties. Here, we summarize recent advances in understanding the mechanisms behind the immune evasion and rice viral pathogenesis. Based on these mechanistic insights, we discuss how to combine different strategies for maintaining the effectiveness of rice resistance to viruses, and propose theoretical basis for the next generation of virus-resistant rice plants.
\end{abstract}

Keywords: Rice virus, Antiviral defense, Pathogenesis

\section{Background}

Rice is the most important crop in Asia and provides staple food for half of the world's population. Rice virus disease causes severe disease of rice in China and many other East Asia countries (Nicaise 2014). Up to now, 17 rice virus diseases have been reported in different areas (Shikata and Kitagawa 1977; Shikata et al. 1979; Omura et al. 1980; Shikata et al. 1980; Xie et al. 1982; Lin et al. 1990; Hibino 1996; Abo et al. 1997; Munoz et al. 2004; Zhou et al. 2008; Yang et al. 2016; Wang et al. 2018), and 6 of them, Southern rice black-streaked dwarf virus (SRBSDV), Rice ragged stunt virus (RRSV), Rice stripe virus (RSV), Rice dwarf virus (RDV), Rice grassy stunt virus (RGSV), and Rice black-streaked dwarf virus (RBSDV) are mainly harmful to rice production (Sasaya et al. 2014). Almost all the rice viruses are transmitted by the insect vectors through persistent or nonpersistent manner and cause some common disease symptoms (Ling 1972). To control the destiny of transmission insect vectors, a large amount of pesticides are used, which lead to huge damage to the environment. Thus,

\footnotetext{
* Correspondence: fengganjianying@163.com; wujianguo81@126.com Vector-borne Virus Research Center, State Key Laboratory of Ecological Pest Control for Fujian and Taiwan Crops, Fujian Province Key Laboratory of Plant Virology, Institute of Plant Virology, Fujian Agriculture and Forestry University, Fuzhou 350002, China
}

(c) The Author(s). 2019 Open Access This article is distributed under the terms of the Creative Commons Attribution 4.0 International License (http://creativecommons.org/licenses/by/4.0/), which permits unrestricted use, distribution, and eases effectively and in an environmentally friendly way.

Rice viruses are obligate intracellular parasites that replicate, express viral proteins and establish infection process in host cell. During their biological cycle, the extensive interactions between viruses and rice host lead to the developmental abnormalities and other phenotypic manifestations that are responsible for viral disease symptoms (Ling 1972). To deeply reveal the viral pathogenic mechanism and excavate resistance genes are critical for controlling rice virus disease. In the last two decades several mechanisms involved in viral pathogenesis have been revealed in rice plants (Zhang et al. 2012; Kong et al. 2014; Rong et al. 2014; Zhang et al. 2016; Zhao et al. 2017). In some cases, a single viral protein can be the main responsible agent of the pathogenesis process (Kong et al. 2014; Rong et al. 2014), while in other cases, several interactions between virus and host factors are needed (Zhu et al. 2005; Jin et al. 2016; Shi et al. 2016; Zhang et al. 2016; Tong et al. 2017; Zhao et al. 2017; Zheng et al. 2017) (Fig. 1). Facing threats, rice plants also employ multiple defense pathways to restrict viral replication and movement, including several classical pathways, such as $N$ genes-mediated 


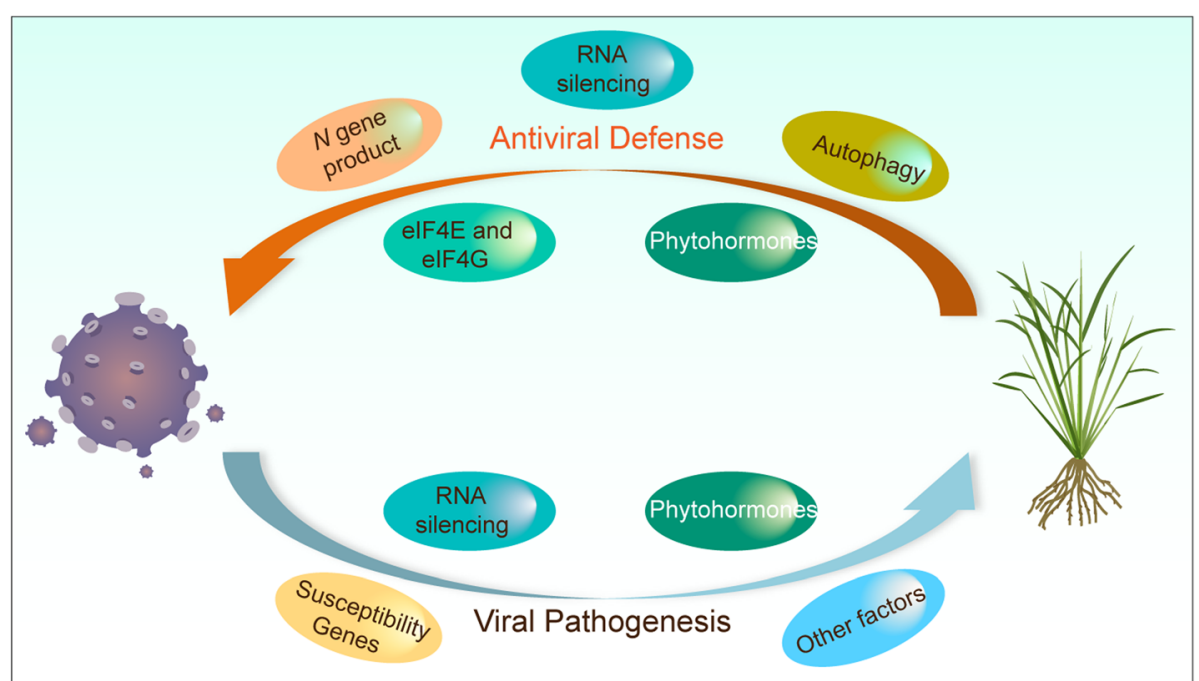

Fig. 1 Schematic diagram showing the arms race between rice host and rice viruses. $N$ gene mediated pathway, RNA silencing pathway, phytohormone pathway, recessive gene resistance and autophagy mediated pathway play important roles during rice-virus interactions

pathway, RNA silencing pathway, phytohormone pathway, recessive resistance genes-mediated pathway and autophagy-mediated pathway (Han et al. 2011; Boualem et al. 2016) (Fig. 1). In recent years, several studies have also provided insights into how defense signaling networks and defense signal molecules intersect with rice-virus interactions (Lee et al. 2010; Wu et al. 2015; Wang et al. 2016; Wu et al. 2017; Yao et al. 2019; Zhang et al. 2019). A deeper understanding of plant antiviral immunity and viral pathogenesis may facilitate innovative biotechnological, genetic and breeding approaches for crop protection and improvement. In this review, we highlight recent discoveries in rice-virus interaction and discuss how these discoveries might inform improved plant protection strategies.

\section{Mechanisms of rice virus pathogenesis}

To establish successful infection, rice viruses induce profound alterations of host physiology, disturbing distinct endogenous processes and contributing to the development of disease. Emerging evidences suggest that virus-encoded proteins display a great variety of functions beyond their canonical roles required for virus structure and replication. Among these functions, their modulation of host immunity appears to be relevant in infection progression.

Symptom formation suggests successful viral replication and pathogenesis. Different rice viruses induce some common viral disease symptoms such as dwarfism, leaf chlorotic specks and sterility. The Rice strip virus (RSV) pc4 has been determined as the viral movement protein (MP) and demonstrated to function in cell-to-cell movement, long distance movement as well as foliar symptom expression in Nicotiana benthamiana (Zhang et al. 2012). Further study proved a transmembrane (TM) domain spanning amino acids 106-123 of pc4 is critical for localization of pc4 to plasmodesmata (PD) and for its ability to recover the movement of movementdeficient PVX (Rong et al. 2014). Another special protein, disease-specific protein (SP) of RSV, has been proved to accumulate in chloroplast, cytoplasm and nucleus in the RSV infected rice cells, and the accumulation level of this protein correlated positively with RSV disease symptom development (Cho et al. 2013). An interesting study found that expression of RSV SP alone in rice or $N$. benthamiana plants does not produce visible phenotypes, but enhances RSV- or Potato virus $X$ (PVX)-induced symptoms through interacting with a $23-\mathrm{kDa}$ oxygen-evolving complex protein, PsbP and disruption of chloroplast structure and function (Kong et al. 2014).

Capsid protein P2 of Rice dwarf virus (RDV) induces disease symptoms by two mechanisms. First, P2 interacts with ent-kaurene oxidases that catalyze a step in the synthesis of gibberellins (GAs), thereby decreasing host GAs levels and promoting the dwarf symptom (Zhu et al. 2005). Second, P2 reprograms the auxin pathway by specifically binding to OsIAA10 and preventing its degradation, leading to enhanced virus infection and the development of typical symptoms including dwarfism, excessive tillering and stunted crown roots in host plants (Jin et al. 2016). Nonstructural protein Pns11 of RDV has been demonstrated to interact with an ethylene synthase, OsSAMS1, and stimulate its activity in triggering 
ethylene production, resulting in elevated susceptibility of plants to RDV (Zhao et al. 2017). These findings indicate that plant hormone pathways are prominent targets for modulation by rice viral proteins. Consistently, a recent study illustrates that jasmonic acid (JA)-mediated defense can suppress the brassinosteroid (BR)-mediated susceptibility to Rice black streaked dwarf virus (RBSDV) infection in rice (He et al. 2017).

The arms race between host and viruses is a continually evolving process involving multiple layers of interactions. Not only plant hormone pathways but also host-microRNA (miRNA) profiles undergo a lot of changes and sometimes they work together against viral invasion. In the case of Rice ragged stunt virus (RRSV) infection of rice, the induced expression of miR319 reduces both the target TCP21 gene expression and JA levels, leading to an elevated plant susceptibility to viral infection (Zhang et al. 2016). MiRNAs serve as an important regulator of plant growth and development by controlling the expression of key developmental and stress-related genes. It therefore comes no surprise that viruses also regulate the expression of host miRNAs. For example, NS3 protein of RSV induces the accumulation of several miRNAs and enhances viral infection and pathogenesis in rice by hijacking OsDRB1, a key component of the microprocessor complex (Zheng et al. 2017). A recent study has demonstrated that reducing the accumulation of miR171b induces RSV-like symptoms in rice whereas miR171b overexpression attenuates RSV symptoms, suggesting that expression of miR171-targeted genes may facilitate RSV infection (Tong et al. 2017).

RNA silencing (also known as RNAi), which acts as a basal defense mechanism against viruses, is now recognized as one of the main plant immune responses against viral pathogens (Guo et al. 2019). As a counter-defense, viruses have also evolved multiple weapons in their armory. Rice RNA-dependent RNA polymerase 6 (RDR6) generates viral double-stranded RNAs for amplifying viral siRNAs (vsiRNAs), which is probably why RDV infection downregulates RDR6 expression. Silencing of RDR6 enhances rice susceptibility to both RDV and RSV infections. However, RDR6 overexpression does not improve rice resistance to RDV infection and RDR6 protein level significantly decreases in RDR6-overexpressing lines after RDV infection, possibly because of translational inhibition of RDR6 and/or destabilization of the protein level by RDV (Jiang et al. 2012; Hong et al. 2015). Furthermore, viral disease symptom such as leaf twisting has been attributed to the vsiRNA-mediated silencing of eukaryotic initiation factor 4A
(eIF4A) in RSV-infected $N$. benthamiana (Shi et al. 2016). Thus, there are multiple aspects of crosstalk between rice and different viruses.

Autophagy can play a pro-microbial role. In turn, pathogens can overcome or even hijack host autophagy for their own benefit (Han et al. 2011). In rice, group 1 remorin (REM1) undergoes S-acylation and is localized in the plasma membrane and plasmodesmata (PD) to inhibit the cell-to-cell movement of viruses. Studies have shown that RSV-encoded NSvc4 can bind to REM1, block its S-acylation, and retain REM1 in the endoplasmic reticulum (ER). Decreased REM1 at PD enables RSV to move to the neighboring cell. The accumulation of non-acylated REM1 at the ER finally triggers autophagy for degradation (Fu et al. 2018).

\section{Multiple layers modulation of rice immunity}

Viruses extensively use host intracellular defense mechanisms for their replication and infection in rice, while plants have evolved intricate defense mechanism depending on a network of signaling pathways involving innate immunity and a class of resistance genes to prevent or limit damage from such pathogens. Base on the recent studies, rice employ three major strategies to counteract virus infection: RNAi-based, plant hormone-mediated and eIF-triggered antiviral defense.

Emerging evidences show that rice viruses induce different subcellular structures in the cytoplasm of infected host cells as the sites of virus replication and packaging. These structures contain many viral proteins important for viral infection, including Pns6, Pns11 and Pns12 of RDV, Pns9 of Rice gall dwarf virus (RGDV), Pc5 of Rice grassy stunt virus (RGSV) and P9-1 of RBSDV and Southern rice black-streaked dwarf virus (SRBSDV). Constitutive expression of transgenes to target these viral genes for RNAi confers resistance against viral infection in transgenic rice plants. However, strong resistance against viral infection has been observed only in transgenic plants targeting some of the viral genes for silencing (Isogai et al. 1998; Wei et al. 2006; Akita et al. 2011; Neme and Tautz 2013; Sasaya et al. 2014). Mechanistic analysis of the natural antiviral RNAi response led to the development of new ideas for transgenic virus resistance in rice (Wu et al. 2015). AGO18 of rice is a monocot-specific AGO protein that sequesters miR168 and prevents its silencing of AGO1, thereby inducing a broadspectrum antiviral RNAi (Wu et al. 2015). AGO18 also sequesters miR528 from AGO1 upon viral infection and prevents miR528 silencing of L-ascorbate oxidase (AO), leading to the elevated accumulation 
of reactive oxygen species (ROS) to enhance antiviral defense (Wu et al. 2017). Further studies showed that OSSPL9 could negatively regulate the expression of antiviral gene AO. OsSPL9 overexpressed plants were more susceptible to the virus, while mutants were more resistant (Yao et al. 2019). Moreover, miR444 expression is triggered by RSV infection to silence the expression of a negative regulator of RDR1 (Wang et al. 2016). These findings suggest that transgenic expression of $A G O 18$ or miR444 may confer broad-spectrum virus resistance in rice.

Phytohormones are for a long time known to act as chemical messengers in the regulation of physiological processes during a whole plant life cycle, from germination to senescence. In addition, phytohormones simultaneously coordinate physiological responses to biotic and abiotic stresses. The intricate network of phytohormone signaling pathways enables plants to activate appropriate and effective defense responses against virus as well as to balance defense and growth. Salicylic acid (SA) was first associated with plant defense through its effects on virus infection and more attention has been paid to the study of SA on plant-virus disease resistance (Palukaitis et al. 2017). SA positively regulates virus resistance and frequently interacts with JA in a mutually antagonistic manner. RESISTANCE TO RSV ON CHROMOSOME 11 (STV11-R) encodes a sulfotransferase protein (SOT1) to catalyze the conversion of SA into sulfonated SA, which inhibits RSV replication. Thus, introgression of the SOT1 allele from Oryza indica and O. japonica rice varieties into susceptible rice cultivars confers strong, durable resistance to RSV (Wang et al. 2014). The NAM/ ATAF/CUC (NAC) transcription factor RIM1 (Rice dwarf virus multiplication 1) is essential for JA signaling and the rim1 mutant exhibits strong resistance to RDV infection (Yoshii et al. 2009; Yoshii et al. 2010), possibly because the SA antiviral response is de-repressed. Auxin is considered as a crucial plant hormone for its role in plant growth and development, also in plant defense against virus (Benjamins and Scheres 2008). A very recent study offered novel insights into the crosstalk regulatory mechanisms of auxin, JA and ROS pathway in rice-RBSDV interaction. Using various auxin signaling mutants, auxin was proved to play a positive role in rice immunity against RBSDV, during this process, JA and ROS pathway also contributed to this defense (Zhang et al. 2019). Thus, it is apparent that the signaling interactions among multiple phytohormones are rather common in rice antiviral defense response.

As invading viruses do not harbor functional ribosomes in their virions, successful replication and infection require cellular translation factors. It is perhaps not surprising that many natural plant recessive resistance genes have been mapped to mutations of translation initiation factors like eIF4G or its isoform eIFiso4G in rice. The natural resistance to a sobemovirus Rice yellow mottle virus (RYMV) conferred by a mutation in eIFiso4G is an interesting case (Albar et al. 2006), since it contains several naturally alleles resistant to RYMV found in O. sativa (rymv12) and O. glaberrima (rymv1-3, rymv1-4, rymv1-5) (Orjuela et al. 2013). Further study proved the interaction between eIF (iso)4G1 and RYMV virulence factor VPg, which correlates with rice susceptibility and RYMV virulence (Hebrard et al. 2010). Another analysis of the corresponding fragments from a number of resistant and susceptible rice varieties suggested that the Rice tungro spherical virus (RTSV) resistance allele $(t s v 1)$ corresponded to eIF4G (Lee et al. 2010). These findings attract us to focus on recessive genesmediated resistance, and its deployment for application in agriculture.

Collectively, these results implicate different ways of RNAi, hormone pathways and recessive resistance genes function in rice antiviral defense, however, from the understanding of plant against virus obtained from work on dicot plants, a complex crosstalk may connect different pathways during immunity, which needs further study in rice.

\section{Conclusions}

We summarizes current knowledge on the riceviruses interaction. It is plausible that different pathways independently or collaboratively fulfil their roles during the arms race between rice and viruses. This inextricable relationship between rice and virus confronts rice plants with challenges that are different in important respects from those presented by other pathogens. The defense and counter-defense between rice and viruses is always on the stage and has many twist and turns (Fig. 2). Despite significant progress in recent years, many aspects of rice-virus relationship remain to be addressed. Where did the rice virus originate? What's the mechanism of intermittent outbreaks of rice virus disease? Are there broad-spectrum immune antigens against rice virus? Understanding the underlying mechanisms will provide the basis for the selection of new sources of resistance. Engineering rice plants with increased resistance to rice viruses is key for ensuring food security and sustainable agriculture. Although the use of genetic resistance is considered to be the most effective and environmentally sustainable strategy for rice virus disease control, its practical use in resistance breeding is still far from reaching a massive scale level. Trade-offs of resistance with other traits can affect a cultivar's overall performance because the aim of plant breeding is not only to improve disease resistance for its own sake but also to produce a cultivar with other desirable agricultural traits. In 


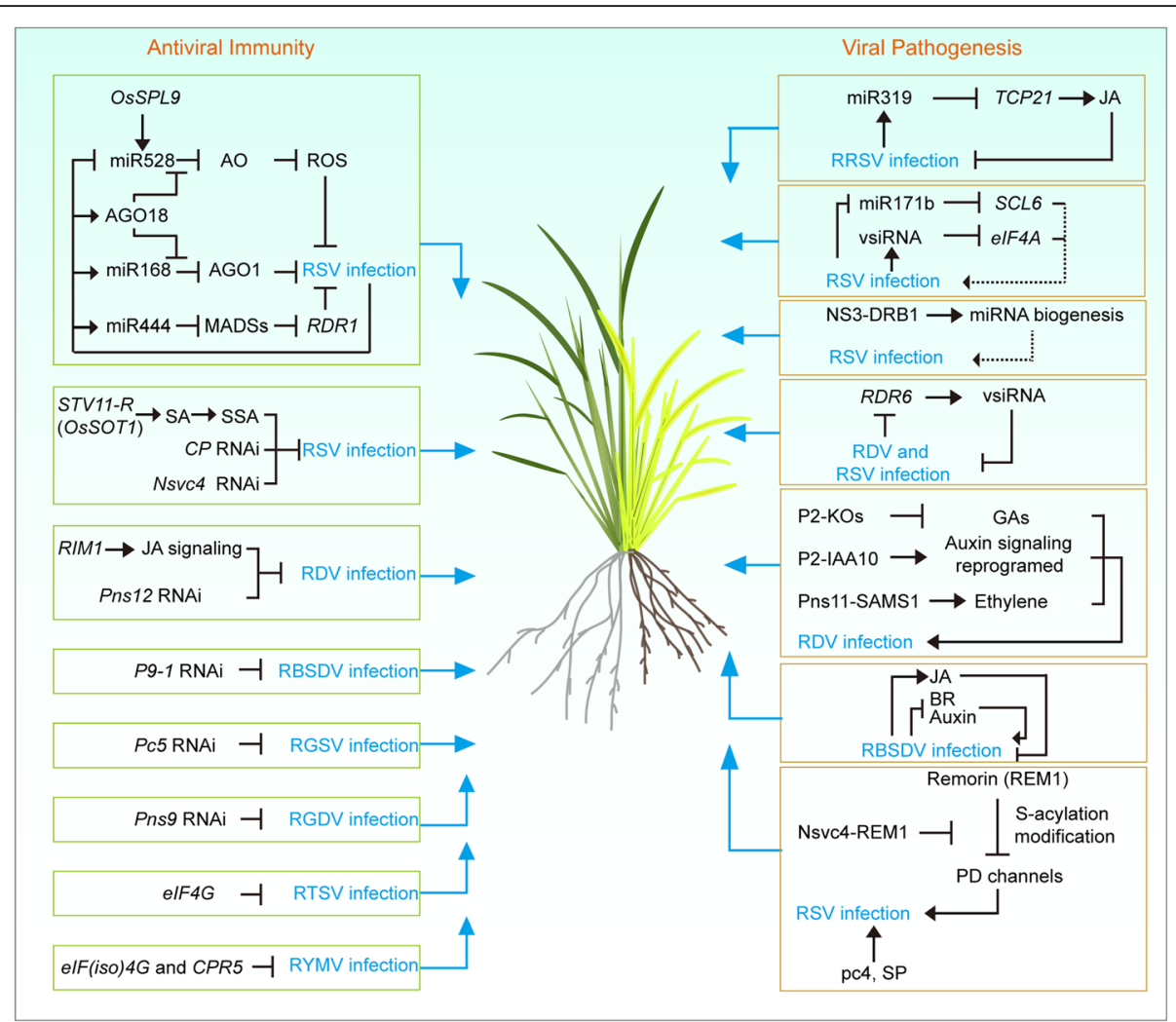

Fig. 2 Mechanisms of rice antiviral defense and viral pathogenesis. A simplified representation of the mechanisms behind rice and viruses agoold battle involving RNA silencing pathway, phytohormone pathway, recessive resistance genes pathway and a subset of miRNAs. The left and right-half plane indicate an overview of different approaches used in rice for antiviral immunity and virial pathogenesis, respectively. Details present in the paper. Note that arrows and lines with bars indicate positive and negative regulatory actions, respectively. RBSDV, Rice blackstreaked dwarf virus; RDV, Rice dwarf virus; RGDV, Rice gall dwarf virus; RGSV, Rice grassy stunt virus; RRSV, Rice ragged stunt virus; RSV, Rice stripe virus; RTSV, Rice tungro spherical virus; RYMV, Rice yellow mottle virus; BR, brassinosteroid; GA, gibberellins; JA, jasmonic acid; SA, salicylic acid

general, plant biotechnologists have focused on engineering broad-spectrum resistant rice varieties in order to keep pace with rapidly evolving rice viruses, and improved tools and methods are becoming available to better integrate different breeding strategies for resistant rice varieties with increased potential in durability.

\section{Abbreviations}

AGO: Argonaute; AGO1: Argonaute1; AGO18: Argonaute18; AO: L-ascorbate oxidase; BR: Brassinosteroid; DRB1: Double-stranded RNA-binding protein 1; elF4A: Eukaryotic initiation factor 4A; ER: Endoplasmic reticulum; GAs: Gibberellins; IAA10: Indole-3-acetic acid 10; JA: Jasmonic acid; miRNA: MicroRNA; MP: Movement protein; NAC: NAM/ATAF/CUC; PD: Plasmodesmata; Pns11: Nonstructural protein 11; PsbP: Extrinsic eukaryotic protein of photosystem II; PVX: Potato virus X; RBSDV: Rice blackstreaked dwarf virus; RDR6: RNA-dependent RNA polymerase 6; RDV: Rice dwarf virus; REM1: Remorin 1; RGDV: Rice gall dwarf virus; RGSV: Rice grassy stunt virus; RIM1: Rice dwarf virus multiplication 1; RNAi: RNA interference; ROS: Reactive oxygen species; RRSV: Rice ragged stunt virus; RSV: Rice stripe virus; RYMV: Rice yellow mottle virus; SA: Salicylic acid; SAMS1: Sadenosylmethionine synthase 1; SOT1: Sulfotransferase protein; SP: Diseasespecial protein; SPL9: Squamosa promoter-binding protein 9;

SRBSDV: Southern rice black streaked dwarf virus; STV11-R: Resistance to RSV on chromosome 11; TCP: Teosinte branched/cycloidea/PCF;

TM: Transmembrane; vsiRNAs: Viral siRNAs

\section{Acknowledgements}

We thank Prof. Shou-Wei Ding (Department of Microbiology and Plant Pathology, University of California, Riverside, CA, USA) for critical reading of the manuscript. Apologies to colleagues whose work could not be discussed due to space limitations. No conflict of interest declared.

\section{Authors' contributions}

JQ, SZ and JW wrote the manuscript. JQ, CW, LW, SZ and JW drew the figures. All authors read and approved the final manuscript.

\section{Funding}

This work was supported by grants from the National Natural Science Foundation of China (No. 31722045, 31871934 and 31772128), Fok Ying-Tong Education Foundation, China (No. 161024), Education and Research Projects for Young Teachers in Fujian Provincial Department of Education (No. JT180112), Outstanding Youth Research Program of Fujian Agriculture and Forestry University (No. XJQ201804).

\section{Availability of data and materials Not applicable.}

Ethics approval and consent to participate Not applicable.

\section{Consent for publication}

Not applicable.

\section{Competing interests}

The authors declare that they have no competing interests. 
Received: 3 October 2019 Accepted: 21 November 2019 Published online: 02 December 2019

\section{References}

Abo ME, Sy AA, Alegbejo MD. Rice yellow mottle virus (RYMV) in Africa: evolution, distribution, economic significance on sustainable rice production and management strategies. J Sustain Agric. 1997;11:85-111.

Akita F, Miyazaki N, Hibino H, Shimizu T, Higashiura A, Uehara-Ichiki T, et al. Viroplasm matrix protein Pns9 from rice gall dwarf virus forms an octameric cylindrical structure. J Gen Virol. 2011;92:2214-21.

Albar L, Bangratz-Reyser M, Hebrard E, Ndjiondjop MN, Jones M, Ghesquiere A. Mutations in the elF (iso) $4 \mathrm{G}$ translation initiation factor confer high resistance of rice to Rice yellow mottle virus. Plant J. 2006;47:417-26.

Benjamins R, Scheres B. Auxin: the looping star in plant development. Annu Rev Plant Biol. 2008;59:443-65.

Boualem A, Dogimont C, Bendahmane A. The battle for survival between viruses and their host plants. Curr Opin Virol. 2016;17:32-8.

Cho WK, Lian S, Kim SM, Park SH, Kim KH. Current insights into research on Rice stripe virus. Plant Pathol J. 2013;29:223-33.

Fu S, Xu Y, Li C, Li Y, Wu J, Zhou X. Rice stripe virus interferes with S-acylation of remorin and induces its autophagic degradation to facilitate virus infection. Mol Plant. 2018;11:269-87.

Guo Z, Li Y, Ding SW. Small RNA-based antimicrobial immunity. Nat Rev Immunol. 2019;19:31-44.

Han S, Yu B, Wang Y, Liu Y. Role of plant autophagy in stress response. Protein Cell. 2011;2:784-91.

He Y, Zhang H, Sun Z, Li J, Hong G, Zhu Q, et al. Jasmonic acid-mediated defense suppresses brassinosteroid-mediated susceptibility to Rice black streaked dwarf virus infection in rice. New Phytol. 2017;214:388-99.

Hebrard E, Poulicard N, Gerard C, Traore O, Wu HC, Albar L, et al. Direct interaction between the Rice yellow mottle virus (RYMV) VPg and the central domain of the rice elF (iso)4G1 factor correlates with rice susceptibility and RYMV virulence. Mol Plant-Microbe Interact. 2010;23:1506-13.

Hibino H. Biology and epidemiology of rice viruses. Annu Rev Phytopathol. 1996; 34:249-74

Hong W, Qian D, Sun R, Jiang L, Wang Y, Wei C, et al. OsRDR6 plays role in host defense against double-stranded RNA virus, Rice Dwarf Phytoreovirus. Sci Rep. 2015;5:11324

Isogai M, Uyeda I, Lee BC. Detection and assignment of proteins encoded by rice black streaked dwarf fijivirus S7, S8, S9 and S10. J Gen Virol. 1998;79:1487-94.

Jiang L, Qian D, Zheng H, Meng LY, Chen J, Le WJ, et al. RNA-dependent RNA polymerase 6 of rice (Oryza sativa) plays role in host defense against negative-strand RNA virus, Rice stripe virus. Virus Res. 2012;163:512-9.

Jin L, Qin Q, Wang Y, Pu Y, Liu L, Wen X, et al. Rice dwarf virus P2 protein hijacks auxin signaling by directly targeting the rice OsIAA10 protein, enhancing viral infection and disease development. PLoS Pathog. 2016;12:e1005847.

Kong L, Wu J, Lu L, Xu Y, Zhou X. Interaction between Rice stripe virus disease-specific protein and host PsbP enhances virus symptoms. Mol Plant. 2014;7:691-708.

Lee JH, Muhsin M, Atienza GA, Kwak DY, Kim SM, De Leon TB, et al. Single nucleotide polymorphisms in a gene for translation initiation factor (elF4G) of rice (Oryza sativa) associated with resistance to Rice tungro spherical virus. Mol Plant-Microbe Interact. 2010;23:29-38.

Lin Q, Xie L, Zhou Z, Xie L, Wu Z. Studies on rice stripe: I. distribution of and losses caused by the disease. J Fujian Agric Coll. 1990:19:421-5.

Ling KC. Rice virus diseases. Los Banos: The International Rice Research Institute; 1972.

Munoz M, Bolanos I, Arrieta-Espinoza G, Espinoza AM. Expression of the rice hoja blanca virus (RHBV) non-structural protein 3 (NS3) in Escherichia coli and its in situ localization in RHBV-infected rice tissues. Rev Biol Trop. 2004;52:765-75.

Neme R, Tautz D. Phylogenetic patterns of emergence of new genes support a model of frequent de novo evolution. BMC Genomics. 2013:14:117.

Nicaise V. Crop immunity against viruses: outcomes and future challenges. Front Plant Sci. 2014;5:660.

Omura T, Inoue H, Morinaka T, Saito Y, Chettanachit D, Putta M, et al. Rice gall dwarf, a new virus disease. Plant Dis. 1980;64:795-7.

Orjuela J, Deless EF, Kolade O, Cheron S, Ghesquiere A, Albar L. A recessive resistance to Rice yellow mottle virus is associated with a rice homolog of the CPR5 gene, a regulator of active defense mechanisms. Mol Plant-Microbe Interact. 2013;26:1455-63.

Palukaitis P, Yoon JY, Choi SK, Carr JP. Manipulation of induced resistance to viruses. Curr Opin Virol. 2017;26:141-8.
Rong L, Lu Y, Lin L, Zheng H, Yan F, Chen J. A transmembrane domain determines the localization of rice stripe virus pc4 to plasmodesmata and is essential for its function as a movement protein. Virus Res. 2014;183:112-6.

Sasaya T, Nakazono-Nagaoka E, Saika H, Aoki H, Hiraguri A, Netsu O, et al. Transgenic strategies to confer resistance against viruses in rice plants. Front Microbiol. 2014:4:409.

Shi B, Lin L, Wang S, Guo Q, Zhou H, Rong L, et al. Identification and regulation of host genes related to Rice stripe virus symptom production. New Phytol. 2016:209:1106-19.

Shikata E, Kitagawa Y. Rice black-streaked dwarf virus: its properties, morphology and intracellular localization. Virology. 1977;77:826-42.

Shikata E, Senboku T, Kamjaipai K, Chou T-G, Tiongco ER, Ling KC. Rice ragged stunt virus, a new member of plant reovirus group. Jpn J Phytopathol. 1979:45:436-43.

Shikata E, Senboku T, Ishimizu T. The causal agent of rice grassy stunt disease. Proc Japan Acad Ser B. 1980:56:89-94.

Tong A, Yuan Q, Wang S, Peng J, Lu Y, Zheng H, et al. Altered accumulation of Osa-miR171b contributes to rice stripe virus infection by regulating disease symptoms. J Exp Bot. 2017;68:4357-67.

Wang Q, Liu Y, He J, Zheng X, Hu J, Liu Y, et al. STV11 encodes a sulphotransferase and confers durable resistance to rice stripe virus. Nat Commun. 2014;5:4768.

Wang $H$, Jiao $X$, Kong $X$, Hamera $S$, Wu $Y$, Chen $X$, et al. A signaling cascade from miR444 to RDR1 in rice antiviral RNA silencing pathway. Plant Physiol. 2016; 170:2365-77.

Wang H, Wang J, Xie Y, Fu Z, Wei T, Zhang XF. Development of leafhopper cell culture to trace the early infection process of a nucleorhabdovirus, rice yellow stunt virus, in insect vector cells. Virol J. 2018;15:72.

Wei T, Shimizu T, Hagiwara K, Kikuchi A, Moriyasu Y, Suzuki N, et al. Pns12 protein of Rice dwarf virus is essential for formation of viroplasms and nucleation of viral-assembly complexes. J Gen Virol. 2006;87:429-38.

Wu J, Yang Z, Wang Y, Zheng L, Ye R, Ji Y, et al. Viral-inducible Argonaute18 confers broad-spectrum virus resistance in rice by sequestering a host microRNA. eLife. 2015:4:e05733.

Wu J, Yang R, Yang Z, Yao S, Zhao S, Wang Y, et al. ROS accumulation and antiviral defence control by microRNA528 in rice. Nat Plants. 2017;3:16203.

Xie L, Lin Q, Zhou Q. On the bunchy stunt disease of rice, II. The distribution, loss, hosts and overwintering of rice bunchy stunt. Acta Phytopathol Sin. 1982; 12(4):16-20 http://zwblxb.magtech.com.cn/CN/abstract/abstract3507.shtml.

Yang X, Huang J, Liu C, Chen B, Zhang T, Zhou G. Rice stripe mosaic virus, a novel Cytorhabdovirus infecting rice via leafhopper transmission. Front Microbiol. 2016;7:2140.

Yao S, Yang Z, Yang R, Huang Y, Guo G, Kong X, et al. Transcriptional regulation of miR528 by OsSPL9 orchestrates antiviral response in rice. Mol Plant. 2019;12:1114-22.

Yoshii M, Shimizu T, Yamazaki M, Higashi T, Miyao A, Hirochika H, et al. Disruption of a novel gene for a NAC-domain protein in rice confers resistance to Rice dwarf virus. Plant J. 2009:57:615-25.

Yoshii M, Yamazaki M, Rakwal R, Kishi-Kaboshi M, Miyao A, Hirochika H. The NAC transcription factor RIM1 of rice is a new regulator of jasmonate signaling. Plant J. 2010;61:804-15.

Zhang C, Pei X, Wang Z, Jia S, Guo S, Zhang Y, et al. The Rice stripe virus pc4 functions in movement and foliar necrosis expression in Nicotiana benthamiana. Virology. 2012;425:113-21.

Zhang C, Ding Z, Wu K, Yang L, Li Y, Yang Z, et al. Suppression of jasmonic acidmediated defense by viral-inducible microRNA319 facilitates virus infection in rice. Mol Plant. 2016:9:1302-14.

Zhang H, Tan X, Li L, He Y, Hong G, Li J, et al. Suppression of auxin signalling promotes rice susceptibility to Rice black streaked dwarf virus infection. Mol Plant Pathol. 2019;20:1093-104.

Zhao S, Hong W, Wu J, Wang Y, Ji S, Zhu S, et al. A viral protein promotes host SAMS1 activity and ethylene production for the benefit of virus infection. eLife. 2017;6:e27529.

Zheng L, Zhang C, Shi C, Yang Z, Wang Y, Zhou T, et al. Rice stripe virus NS3 protein regulates primary miRNA processing through association with the miRNA biogenesis factor OsDRB1 and facilitates virus infection in rice. PLoS Pathog. 2017;3:e1006662.

Zhou G, Wen J, Cai D, Li P, Xu D, Zhang S. Southern rice black-streaked dwarf virus: a new proposed Fijivirus species in the family Reoviridae. Chin Sci Bull. 2008; 53:3677-85.

Zhu S, Gao F, Cao X, Chen M, Ye G, Wei C, et al. The rice dwarf virus P2 protein interacts with ent-kaurene oxidases in vivo, leading to reduced biosynthesis of gibberellins and rice dwarf symptoms. Plant Physiol. 2005;139:1935-45. 